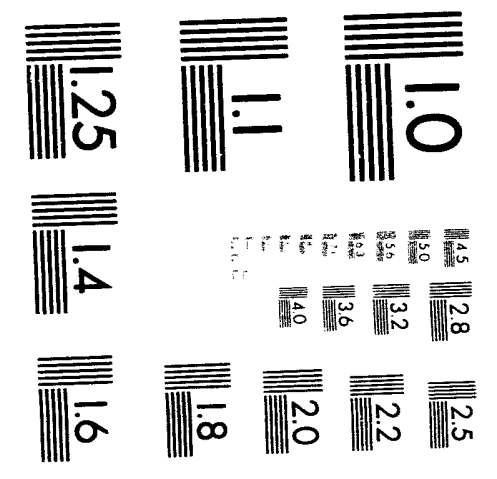



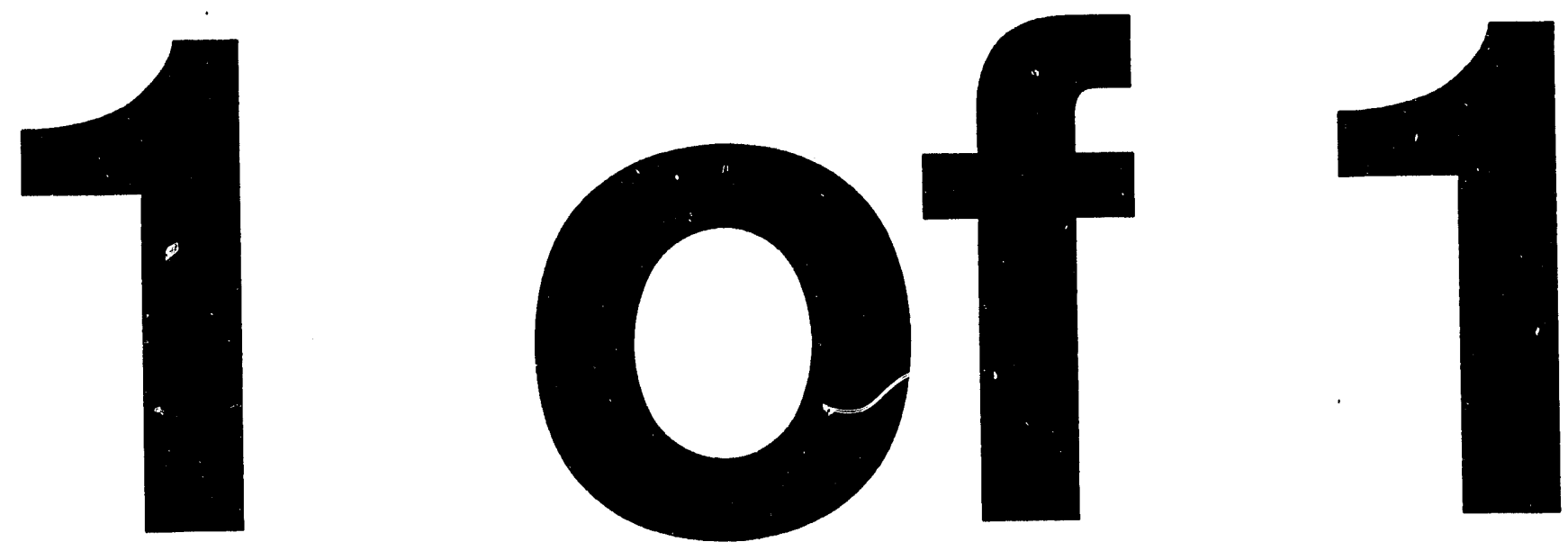


\section{DISORDER EFFECTS ON \\ SMALL-POLARON FORMATION AND HOPPING

$\begin{array}{cl}\text { David Emin } & \begin{array}{l}\text { All papers must include the followin } \\ \text { statement: }\end{array} \\ \text { Sandia National Laboratories } & \begin{array}{l}\text { This work performed at Sandia National } \\ \text { Laboratories is supported by the }\end{array} \\ \text { Albuquerque, New Mexico, 87185 USA } & \begin{array}{l}\text { U.S. Department of Encrgy under } \\ \text { contract OE. ACO4. } 960 \text { P00189. }\end{array}\end{array}$

Introduction

Questions concerning effects of disorder on electronic transport in solids have received considerable attention for several decades. In most instances disorder is thought to produce variations of electronic energies that are only a small fraction of the electronic bandwidth, 2W.1,2) By themselves, energy variations of this magnitude only introduce a small density of localized states in the tails of the electronic energy bands. These bandtail localized states are analogous to shallow-impurity states of doped semiconductors. In analogy with doped crystalline semiconductors, steady-state transport above very low temperatures is expected to be dominated by motion of quasifree carriers associated with "extended states." In brief, this picture views amorphous semiconductors as being akin to doped crystalline semiconductors.

Nonetheless, steady-state Hall effect measurements in the vicinity of room temperature find mobilities that are very low $\left(\ll 1 \mathrm{~cm}^{2} / \mathrm{V}-\mathrm{sec}\right)$ and thermally activated. ${ }^{3)}$ Mobilities with these magnitudes and temperature dependences are incompatible with the quasifree carriers expected of extended states. Hall effect measurements also diverge qualitatively from expectations of extended-state motion in that the signs of the Hall effect are often found to be opposite to those determined from thermoelectric power measurements.4) In addition, standard analyses of the temperature dependences of the $\mathrm{dc}$ conductivities and thermoelectric powers in the vicinity of room temperature imply that the carriers' mobilities are thermally activated with activation energies of several tenths of an eV. ${ }^{3)}$ These activation energies are much larger than those associated with hopping between shallow-impurities $\left(10^{-4}\right.$ to $\left.10^{-3} \mathrm{eV}\right)$. Thus, steady-state electronic transport in amorphous semiconductors is qualitatively different from that of doped crystalline semiconductors.

It was noted some time ago that many properties of amorphous semiconductors could be explained if the charge carriers self-trapped to form small polarons. $3,5,6)$ Self trapping occurs when an electronic carrier lingers at a site long enough to permit surrounding atoms to displace in response to the 


\section{DISCLAIMER}

This report was prepared as an account of work sponsored by an agency of the United States Government. Neither the United States Government nor any agency thereof, nor any of their employees, makes any warranty, express or implied, or assumes any legal liability or responsibility for the accuracy, completeness, or usefulness of any information, apparatus, product, or process disclosed, er reprezents that its use would not infringe privately owned rights. Reference herein to ary specific commercial product, process, or service by trade name, trademark, manufacturer, or otherwise does not necessarily constitute or imply its endorsement, recommendation, of favoring by the United States Government or any agency thereof. The views and opinions of authors expressed herein do not necessarily state or reflect those of the United States Government or any agency thereof. 
presence of the carrier. The electronic carrier then becomes severely localized within a potential well produced by the atomic displacements. This "selftrapped" carrier cannot move unless the atoms alter their positions. The localized carrier together with the atomic displacement pattern that confines it is termed a small polaron. The adjective "small" denotes the severe localization of the electronic state. The energy of a small polaron in a covalent solid is lower than that of a static electron by $E_{b} \equiv F^{2} / 2 k$, where $F$ is the force between the carrier and atoms adjacent to it, and $k$ is the material's stiffness constant. As a result of its severe localization, a small polaron generally moves by phonon-assisted hopping.

Small-polarons will only form in covalent crystals whose electronic halfbandwidths are sufficiently narrow, $\left.E_{b}>W .7,8\right)$ The absence of small polaronic carriers in most covalent crystals presumably indicates that $E_{b}<W$ in these instances. However, evidence of small polarons is commonly found in disordered materials despite the estimates of $E_{b}$ and $W$ not being significantly different from those of crystals. This result is rationalized by stating that disorder has slowed carrier motion enough to permit small-polaron formation. $3,5,6$ ) Recently the question of how disorder affects the stability of quasifree (extendedstate) carriers with respect to small-polaron formation has been addressed.9) It is found that only modest energetic disorder is required to induce small-polaron formation. Here I first succinctly describe essential elements of this work.

Second, I address the role of disorder on the adiabatic hopping motion of small polarons. Energy bands in most materials in which small-polarons are found are thought to be sufficiently wide ( $>$ a phonon energy) that the smallpolaronic hopping is "adiabatic."10,11,12,13) That is, the electronic carriers move between sites sufficiently rapidly to follow the atomic motions. In this situation the small-polaron jurnp rates are independent of intersite separations. The magnitudes of the preexponential factors of the measured hopping mobilities typically support this view.3) Further support for this picture is found from experiments that determine weak dependences of the mobility on hydrostatic pressure. ${ }^{14,15)}$

With adiabatic hopping, phenomena that are often interpreted as arising from strong dependences of the jump rate on intersite separation must be reinterpreted. For example, when hopping is adiabatic the nonArrhernius fall of the hopping mobility with decreasing temperature cannot be due to variablerange hopping. However, nonArrhenius temperature dependences of the hopping mobility, observed in crystals as well as noncrystalline systems, also result from the "freeze out" of multiphonon jump processes. ${ }^{16,17)}$ In this paper the very slow dispersive hopping that produces the frequency dependence of the 
conductivity at low frequencies and temperatures is addressed.18) With adiabatic small polaronic hopping, the very slow hops that dominate the low-frequency response occur between sites that are especially close to one another.

\section{Small-polaron formation in a disordered covalent solid}

The nature of a self-trapped carrier depends critically on the range of the electron-lattice interaction.7,19) For the short-range electron-lattice interaction that characterizes covalent materials (the deformation-potential or the Holstein interaction) ${ }^{20)}$ the only type of self-trapped carrier that can form is a small polaron. By contrast, the long-range electron-lattice interaction of ionic systems supports the formation of "large" polarons. While the self-trapped carrier of a small polaron is confined to a single site, the self-trapped carrier of a large polaron generally extends over several sites. In this work, only small-polaron formation in covalent systems is considered.

Small-polaron formation in disordered covalent solids is addressed by considering the groundstate of a carrier added to a disordered insulator composed of displaceable atoms.9) The groundstate wavefunction is expressed as the generalized tight-binding expansion:

$$
\Psi=\sum_{g} b_{g}|g\rangle\left|\chi_{g}\right\rangle
$$

Here $|g\rangle$ is a Wannier function centered at site $g$ and $\left|\chi_{g}\right\rangle$ represents the wavefunction for atomic vibrations when the carrier occupies site $g$. In the absence of the electron-lattice interaction $\left|\chi_{g}\right\rangle$ is independent of the site occupied by the carrier. When the electron-lattice interaction is sufficient so that carriers form small polarons $\left|\chi_{g}\right\rangle$ describes the vibrations of atoms about the displaced equilibrium positions they assume when the carrier is held at site $\mathbf{g}$.

The groundstate energy is expressible in terms of variational parameters, the $X_{g}$ 's, that are the shifts of the equilibrium separation of the bond occupied by the carrier. The energy of the system when a carrier resides on site $g$, is then

$$
E_{g}=k\left(X_{g}\right)^{2} / 2-F X_{g}-\sum_{h} t_{g} g+h \exp \left\{-k\left[\left(X_{g}\right)^{2}+\left(X_{g}+h\right)^{2}\right] / 4 h v\right\},
$$

where $\mathbf{k}$ is the solid's stiffness constant, $\mathrm{F}$ is the force between a carrier on the $\mathbf{g}$-th bond and the atomic displacement coordinate associated with that bond, $t_{g, g+h}$ is 
the electronic transfer energy between the $\mathrm{g}$-th bond and that of a neighboring bond labelled by $g+h$, and $v$ is the characteristic vibrational energy. The first contribution to the energy of Eq. (2) is the strain energy associated with stretching the bond at site $g$ by $X_{\mathbf{g}}$. The second contribution in Eq. (2) is the lowering of the electronic energy of a carrier at site $g$ as a result of expanding the $g$-th bond. The final term is the net transfer energy associated with the carrier's motion from site $\mathbf{g}$ to neighboring sites labelled as $\mathbf{g}+\mathbf{h}$. Altering the bond length of sites $\mathbf{g}$ or $\mathbf{g}+\mathbf{h}$ is seen to reduce the lowering of the energy associated with the motion of a carrier to an adjacent site.

The groundstate energy is found by minimizing each $E_{g}$ with respect to the $X_{\mathbf{g}}$. Carrying out this minimization procedure results in a set of coupled nonlinear equations whose solution gives the $X_{g}$ 's:

$$
X_{g}=\frac{F / k}{1+\sum_{h}\left(t_{g} ; g+h / h v\right) \exp \left[-k\left[\left(X_{g}\right)^{2}+\left(X_{g}+h\right)^{2}\right] / 4 h v\right]^{-}}
$$

The minimization procedure yields either the small-polaron or free-carrier solution at a site. In the adiabatic limit, $v \rightarrow 0$, the small-polaron solution is $X_{\mathbf{g}}=$ $F / k$ with $E_{g}=-E_{b}$. The free-carrier solution is then $X_{\mathbf{g}}=0$ with $E_{\mathbf{g}}=-W_{\mathbf{g}}$, where the local "halfbandwidth" is $W_{g} \equiv \sum_{h} t_{g} ; g+h$ with the summation now only extending over sites at which the free-carrier state is stable: $W_{g}>E_{b}$. The limiting of the sites over which the summation extends is a manifestation of the nonlinearity of the self-trapping process. This "feedback" effect enhances the disorder's fostering of self-trapping.

A probabilistic approach is used to discuss disorder-induced small-polaron formation. In particular, first presume a distribution of electronic transfer energies, $\rho(t)$, that permits smaller values of the $t$ in a disordered material than that characterizing a crystal, to. Then the probability of a site being one at which small-polaron formation is stable is written as:

$$
P_{s p}=\int_{0}^{E_{b} /\left(z-z_{s p}\right)} d t \rho(t)=\int_{0}^{E_{b} / z\left(1-P_{s p}\right)}
$$

where $z$ is the total number of nearest neighbors and $z_{s p}$ is the number of nearest neighbors at which small-polaron formation is stable. To obtain the final equality of Eq. (4), a mean-field approach is adopted: $z_{s p}=z P_{s p}$. Figure 1 shows $P_{s p}$ plotted 
against $E_{b} / z t_{0}$ for one model of $\left.\rho(t) .9\right)$ For the crystal, the dashed lines, small polarons are only stable when $E_{b} / z t_{0}>1$. As the spread of an asymmetric distribution of transfer energies is increased, curves $a, b, c$ and $d$ are successively obtained. These curves show global small-polaron formation, $P_{s p}=1$, occurring at successively smaller values of $\mathrm{E}_{\mathrm{b}}$ as disorder increases.



Fig. 1. $P_{s p}$ is plotted against the $E_{b} / z t_{c}$ for four $(a \rightarrow d)$ successively broader exponential distributions of transfer energies. The dashed curve is for a crystal with transfer energy $t_{0}$. For curve $d$ the average transfer energy has fallen to $t_{0} / 2$.

In summary, a carrier can only exist without forming a small polaron if it moves between sites quickly enough. By impeding a carrier's motion, disorder fosters small-polaron formation. In particular, sites at which small polaron formation is stabilized cannot be used in paths over which a carrier is moving freely. This "feedback" effect enhances disorder's tendency to foster smallpolaron formation. Therefore, even modest disorder, disorder energies much less than the electronic bandwidth, can stabilize small-polaron formation in disordered covalent materials. 
Low-temperature low-frequency conductivity for adiabatic small-polaron hopping

Since the electronic carrier of a small polaron is bound by atomic displacements, the carrier can only move when the atoms alter their positions. A hop of a charge carrier between sites requires atoms to undergo displacements that are much larger than the zero-point amplitude: $F / k>\sqrt{h v / k .10)}$

At sufficiently high temperatures, typically $k T>h v / 3$, such large amplitude vibrations ( $>\mathrm{F} / \mathrm{k}$ ) occur often enough that a substantial hopping rate is achieved when the electronic carrier follows classical atomic motion. In this regime, the small-polaron jump rate, $R_{\text {hop, }}$ is thermally activated: $R_{\text {hop }}=v \exp \left(-E_{a} / k T\right) P$, where $P$ is the probability that the carrier follows the atomic motion. 5,10$)$ Here $E_{a}$ is the minimum strain energy required to move atoms into a configuration (termed a coincidence configuration) through which the carrier transfers between sites. In the adiabatic regime, where the carrier is assumed to follow the atomic motion, $P \approx 1$. However, if the electronic transfer energy, $t$, is so small that the carrier cannot follow classical atomic motion, $\alpha_{h} \equiv(2 \pi)^{3 / 2} t / \sqrt{4 E_{b} \sqrt{h v k T}}<1$, the carrier's motion is limited by the electronic overlap and $P \approx \alpha^{2}$. Estimates of these parameters and experimental evidence both indicate that small-polaron hopping is usually adiabatic. ${ }^{10-15)}$ Nonetheless, because of the relative ease of nonadiabatic calculations, most theoretical studies presume nonadiabaticity.

At low enough temperatures, these large-amplitude classical motions become so infrequent that hopping motion is dominated by a process that requires atoms to tunnel between different configurations. Since the tunneling of atoms through distances greater than the zero-point amplitude is itself rare, the low- temperature small polaron jump rate becomes very small even for hops that are adiabatic [with $t>\sqrt{E_{b} h v / \pi}$ at low temperatures]. ${ }^{10}$ ) In this low-temperature regime, the jump rate falls in a nonArrhenius manner with decreasing temperature as thermal vibrations are progressively frozen out. 16

The effects of disorder on hopping conduction increase progressively as the temperature is reduced. Namely, in a medium with energetic disorder only hops among sites within the thermal energy, kT, of the chemical potential are thermally accessable. At low enough temperatures these sites will be contained within bounded spatial regions of "radius" R, "polarization centers." Carriers remaining in these regions avoid making very energetically unfavorable hops.

For carriers to contribute to dc conduction, they must leave these regions. However, some carriers can contribute to the ac conductivity while remaining in polarization centers. This polarization conductivity may be written as $\sigma_{\text {pol }}(\omega)=$ 
$\mathrm{n}(\omega, \mathrm{T}) \mathrm{q}\left[(\mathrm{q} / \mathrm{kT}) \omega \mathrm{R}^{2}\right]$, where the square-bracketed term is the "mobility" of carriers that relax at the rate $\omega$. This equation may be regarded as a definition of the density of polarization centers whose carriers relax at a frequency near $\omega, n(\omega, T)$.

For definiteness, consider polarization centers as close pairs of sites with energies that straddle the chemical potential. Then $n(\omega, T)=\left\langle\rho p o l(\Delta) P_{p}(\Delta, T)\right.$ $R(\Delta, \omega)>$, where the average is performed over pairs of sites. Here $\rho$ pol $(\Delta)$ is the density of pairs having the energy separation $\Delta_{;} P_{p}(\Delta, T)$ is a pair's polarizability at the temperature $T$; and $R(\Delta, \omega)$ is the dynamic response of a carrier on these sites at the frequency $\omega$. Explicitly, $R(\Delta, \omega) \equiv 2 \omega \tau(\Delta) /\left(1+[\omega \tau(\Delta)]^{2}\right]$ peaks at $\omega \tau=1$.

In disordered systems with adiabatic small-polaron hopping a polarization conductivity is generally observed at very low frequencies (often $10^{2}$ to $10^{6} \mathrm{~Hz}$ ) at low temperatures ( $<25 \mathrm{~K}$ in boron carbides). 13,21) This polarization conductivity is slightly less than linearly proportional to the applied field.

Several significant questions arise in applying the general formalism for the polarization conductivity to these experiments. First, why are the hopping rates so slow, $\approx \omega$ ? Indeed, the small intersite separations, $<10 \AA$, in boron carbides and transition-metal oxide glasses constrain the hops to be rather short. Second, the near-linear dependence of the polarization conductivity on $\omega$ arises from having sufficient dispersion of the hopping rates. What aspect of adiabatic hopping produces this dispersion?

To address these questions, consider the low-temperature relaxation rate for a pair of sites with energy separation $\Delta:{ }^{18)}$

$$
1 / \tau=v\left[\left(E_{b} / h v\right) \exp \left(-E_{b} / h v\right)\right]^{2}(\Delta / h v)^{3} P
$$

For adiabatic small-polaron hops: $E_{b} / h v \gg 1$ and $P=1$. The factor $\exp \left(-2 E_{b} / h v\right)$ arises from the atomic tunneling required for low-temperature small-polaron hops. The smallness of this low-temperature relaxation rate is primarily due to the smallness of this factor. For example, the square of the square-bracketed term is $10^{-8}$ for typical numbers, $\mathrm{E}_{\mathrm{b}} / \mathrm{hv}=10$. By contrast, the analogous expression for nonadiabatic hops between widely separated shallow impurities lacks the factor $\exp \left(-2 E_{b} / h v\right)$ but has $P \ll 1$. Thus, the smallness of the relaxation rate for shallow-impurity conduction is mainly caused by the large separation between impurities.

For low-temperature small-polaronic hops, dispersion of the relaxation rates results from their proportionality to $(\Delta / \mathrm{hv})^{3}$, where $\Delta \ll \mathrm{hv}$. This factor occurs because the wavelengths of emitted phonons generally exceed the jump 
distance. However, for shallow-impurity conduction with jump distances exceeding the wavelengths of emitted phonons, the comparable factor is $\Delta \mathrm{hv}$.

The relaxation becomes especially slow for hops between nearly degenerate states, $\Delta \rightarrow 0$. Since two sites that are especially close to one another share a common "external" environment, they are nearly degenerate. Thus, especially short small-polaronic hops can be the slowest. By contrast, very slow hops between shallow-impurities are unusally long.

Incorporating the relaxation rate of Eq. (5) into the previously described formalism yields an expression for $\sigma_{\text {pol }}(\omega)$. In the limit of $T \rightarrow 0$, $\sigma_{\text {pol }}(\omega) \rightarrow \omega T$. Above this low-temperature limit, the severity of the temperature dependence increases with increasing temperature. The temperature dependence in this higher temperature regime also weakens with increasing frequency. These results, obtained in Ref. (18), can be seen in Fig. 2. The curves are in reasonable agreement with experimentally obtained curves.21,22)

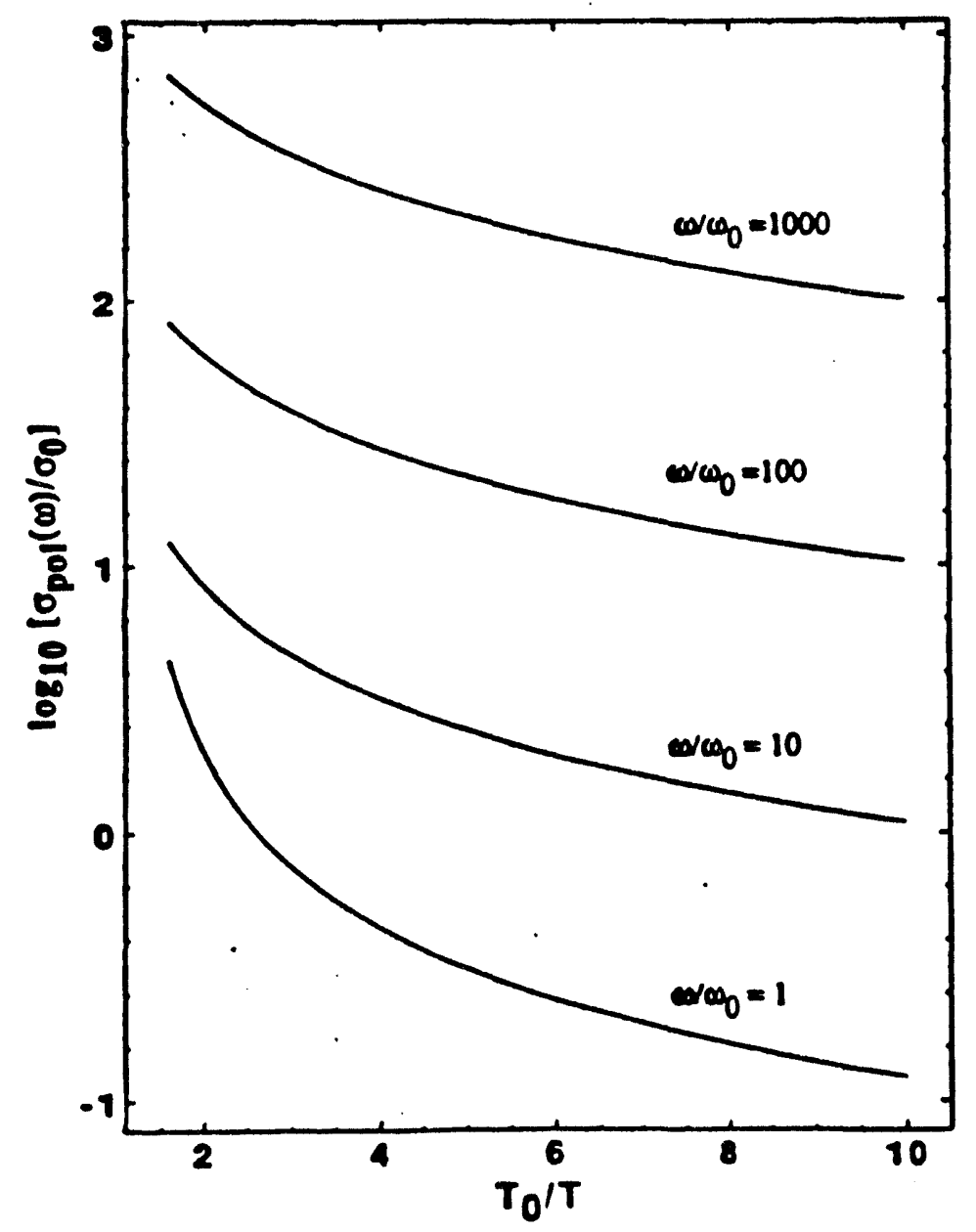

Fig. 2. Normalized polarization conductivity versus a dimensionless reciprocal temperature for four reduced frequencies. 


\section{References}

1. Cohen, M. H., Fritzsche, H., Ovshinsky, S. R. , Phys. Rev. Lett. 22, 1065 (1969).

2. Mott, N. F., Adv. Phys. 16, 49 (1976).

3. Emin, D., Seager, C. H. and Quinn, R. K., Phys. Rev. Lett. 28, 813 (1972).

4. Emin, D., Phil. Mag. 351188 (1977).

5. Emin, D. in Electronic and Structural Properties of Amorphous Semiconductors, edited by P. G. LeComber and J. Mort (Academic, New York, 1973), pp. 262-328.

6. Emin, D., in Electrical and Optical Properties of Amorphous Thin Films, edited by L. Kazmerski (Academic, New York, 1980) pp. 17-57.

7. Toyozawa, Y., Prog. Theor. Phys. 26,29 (1961).

8. Emin, D., Adv. Phys. 22,57 (1973).

9. Emin, D. and Bussac, M.-N., Phys. Rev. B (submitted).

10. Holstein, T., Ann. Phys. (N.Y.) 8,343 (1959).

11. Herring, C., Proc. Int. Conf. on Semiconductor Physics Prague 1960 (Academic Press, New York 1961) pp. 60-67.

12. Emin, D. and Holstein, T., Ann. Phys. (N.Y.) $\underline{53} 439$ (1969).

13. Austin, I. G. and Mott, N. F. Adv. Phys. 18,41 (1969).

14. Dolezalek, F. K. and Spear,W. E., J. Noncryst. Solids 497 (1970).

15. Samara, G. A., Emin, D. and Wood, C., Phys. Rev. B 32, 2315 (1985).

16. Emin, D., Phys. Rev. Lett. 32,303 (1974).

17. Zuppiroli, L. and Forro, L., Phys. Lett. A 141, 181 (1989).

18. Emin, D. Phys. Rev. B 46,9419 (1992).

19. Emin, D. and Holstein, T., Phys. Rev. Lett. $\underline{36} 323$ (1976).

20. Holstein, T., Ann. Phys. (N.Y.) 8325 (1959).

21. Zuppiroli, L., Papandreou, N. and Kormann, R., J. Appl. Phys. 70246 (1991).

22. Samara, G. A., H. L. Tardy, Venturini, E. L., Aselage, T. L., and Emin, D., Phys. Rev. B 48,1468 (1993). 

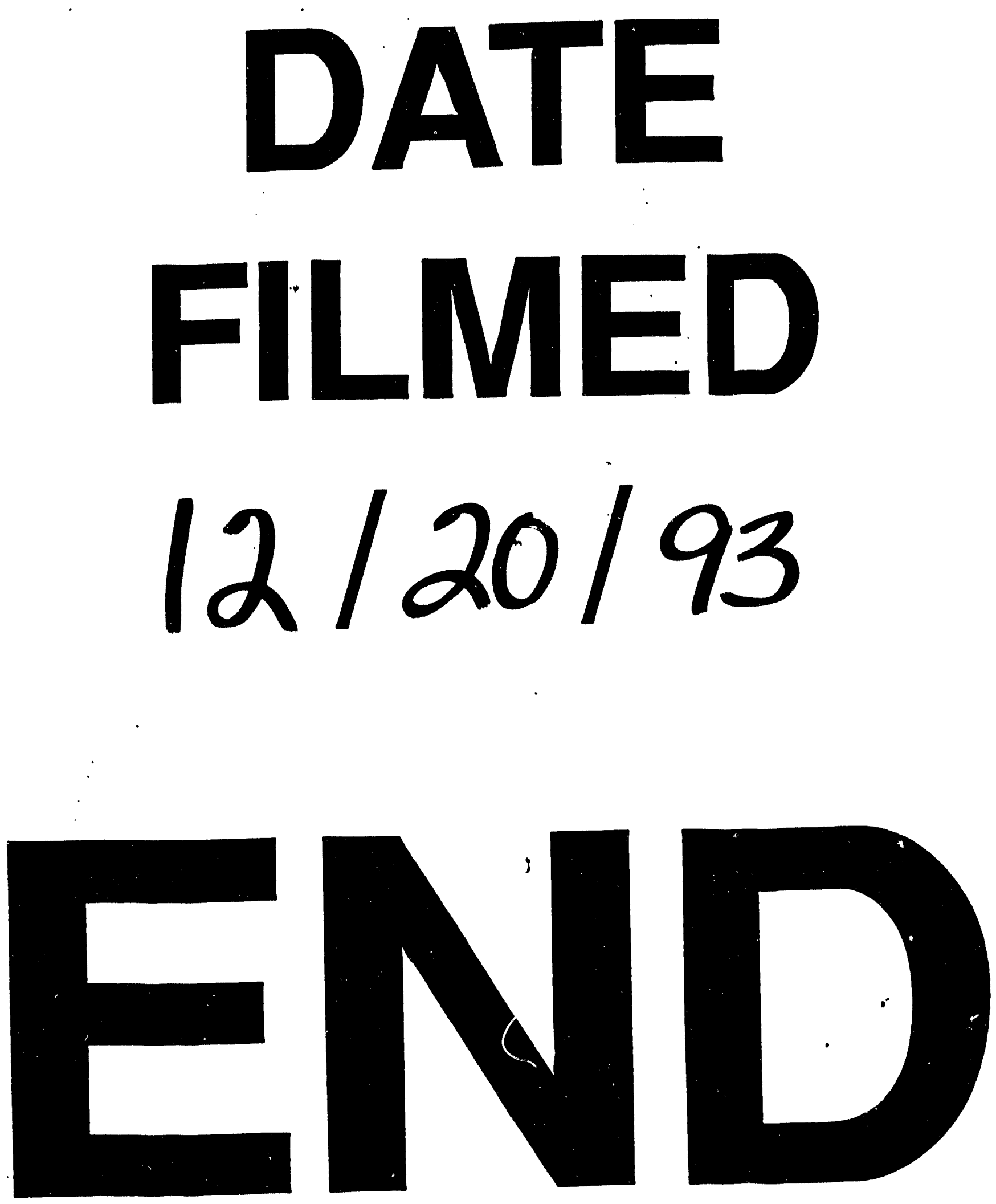